\title{
Expanded Constellation Mapping for Enhanced Far-End-Cross-Talk Cancellation in G.fast
}

\author{
Rong Zhang ${ }^{1}$, Anas F. Al Rawi ${ }^{2}$, Leslie Derek Humphrey ${ }^{2}$, and Lajos Hanzo ${ }^{1}$ \\ ${ }^{1}$ Southampton Wireless, School of ECS, University of Southampton, SO17 1BJ, UK \\ ${ }^{2}$ Research and Technology, British Telecom (BT), Adastral Park, Martlesham Heath, IP5 3RE, UK \\ Email: rz@ecs.soton.ac.uk, http://www-mobile.ecs.soton.ac.uk
}

\begin{abstract}
We propose the novel concept of Expanded Constellation Mapping (ECM) for maximising the received signal power, while cancelling the Far-End-Cross-Talk (FEXT) in copper based wireline communications. The goal of ECM is to beneficially map the transmitted symbol vector to its expanded constellation set by carefully exploiting the copper channels' specific characteristics. To elaborate, ECM is comprised of the control entity and of the match entity, where the former determines how ECM would be applied, while the latter searches for the best mapping of the transmitted symbol vector to the expanded constellation set. Our numerical results demonstrate that with the aid of ECM, more than $25 \mathrm{~dB}$ power efficiency gain may be achieved over linear vectoring. Similarly, about $20 \mathrm{~dB}$ gain may be achieved over nonlinear vectoring. From an implementation point of view, ECM imposes minimal structural changes on G.fast, whilst exhibiting beneficial reconfigurability and compatibility.
\end{abstract}

\section{INTRODUCTION}

To fulfil the ambition of $\mathrm{Gb} / \mathrm{s}$ speeds over copper, the latest Digital Subscriber Line (DSL) standard of G.fast [1], [2] further extends the copper bandwidth up to around 200 MHz. However, this bandwidth expansion inevitably increases the Far-End-Cross-Talk (FEXT) between copper lines. Hence, linear vectoring is employed in G.fast to pre-compensate the downstream FEXT [3]. Unfortunately, the FEXT becomes even higher than the received signal power at higher frequencies. In this scenario, applying linear vectoring significantly reduces the useful received signal power, when precompensating the detrimental FEXT, hence leading to an eroded performance.

A promising technique of improving linear vectoring is the non-linear vectoring [4], which relies on applying mathematical transformations so that the first copper line is free from FEXT and any other copper line is only affected by the FEXT from its previous copper lines. Hence, instead of completely eliminating the FEXT as in linear vectoring, nonlinear vectoring aims for recursively pre-cancelling the FEXT. Nevertheless, several drawbacks exist in non-linear vectoring. Structurally, non-linear vectoring is not backwards compatible with the linear vectoring module of G.fast. Algorithmically, the attainable performance of non-linear vectoring heavily depends on the specific ordering during the successive FEXT decontamination of the copper lines.

Against this background, we propose the novel concept of Expanded Constellation Mapping (ECM), which aims for maximising the received signal power, while still maintaining perfect FEXT pre-cancellation as in linear vectoring, at a

The financial support of the two EPSRC projects (EP/N004558/1 and EP/N023862/1) and that of the European Research Council's (ERC) Advanced Fellow Grant is gratefully acknowledged. minimal structural change imposed on G.fast. In the following, we formulate the problem in Section II and provide the proposed solution in Section III. Our numerical results are discussed in Section IV and we finally conclude in Section V.

\section{Problem Description}

Let us firstly introduce the principle of linear vectoring and its deficiencies. The DSL system is operated on a tone-bytone basis, relying on the so-called Digital Multi-Tone (DMT) technique. Our forthcoming elaborations will be based on a particular tone, but for simplicity we dropped the tone index without loss of generality. Explicitly, on a particular tone, the downstream system can be described as $\boldsymbol{y}=\boldsymbol{H} \boldsymbol{s}+\boldsymbol{n}$, where $\boldsymbol{H}$ represents the channel matrix of $K$ copper lines, characterising the input and output relationship between the $K$ transmitted symbols $s$ at the Distribution Point Unit (DPU) side and the $K$ received symbols $\boldsymbol{y}$ at the Network Termination (NT) side. Furthermore, $\boldsymbol{n}$ represents the Additive White Gaussian Noise (AWGN) with each entry having a zero mean and a variance of $\sigma^{2}$, which is independent of the transmitted symbols.

It is widely recognised that the off-diagonal entries of the copper channel matrix $\boldsymbol{H}$ generate FEXT, which may be pre-cancelled at the transmitter with the aid of linear vectoring by multiplying the transmitted symbols $s$ with the inverse of the copper channel matrix $\boldsymbol{H}$ at the DPU-side. Mathematically, we transmit $\boldsymbol{x}=\sqrt{1 / \beta} \boldsymbol{H}^{-1} \boldsymbol{D} \boldsymbol{s}$, where $\boldsymbol{D}$ is a diagonal matrix, representing the conventional Zero-Forcing (ZF) based linear vectoring when $\boldsymbol{D}=\boldsymbol{I}$ and the Diagonalising based linear vectoring when $\boldsymbol{D}=\operatorname{diag}(\boldsymbol{H})$. Furthermore, $\beta=\mathbb{E}_{\boldsymbol{s}}\left[\left\|\boldsymbol{H}^{-1} \boldsymbol{D} \boldsymbol{s}\right\|^{2}\right]$ is the normalisation factor introduced for avoiding transmit power amplification after pre-cancelling the FEXT ${ }^{1}$, although we can alternatively impose a more strict instantaneous normalisation. Consequently, the downstream system model becomes

$$
\boldsymbol{y}=\boldsymbol{H} \boldsymbol{x}+\boldsymbol{n}=\sqrt{1 / \beta} \boldsymbol{D} \boldsymbol{s}+\boldsymbol{n},
$$

which is now completely free from FEXT, albeit this is achieved at the cost of reducing the received signal power by a factor of $\beta$.

Conventional method to alleviate the received signal power reduction requires the design of an extra beamforming vector [5]. However, this approach would violate the condition for maintaining complete FEXT cancellation. Hence, we treat the

\footnotetext{
${ }^{1}$ Although sum power constraint for $K$ copper lines is considered here, our discussion is also applicable to per-line power constraint with its performance included in Section IV. However, the optimal power allocation obeying perline power constraint for $K$ copper lines will need further investigation.
} 
transmitted symbol vector as the beamforming vector, where the design degree-of-freedom comes from the mapping of the transmitted symbols to their expanded constellation sets. This is true, since any integer expansion on symbol constellations made at the transmitter may be recovered by simple modulo operation at the receiver. In this way, the received signal power reduction is minimised on a per transmitted symbol vector basis, which guarantees the minimisation on $\beta$. As an example, let $\boldsymbol{H}^{-1}=[1,1 ; 0.5,1]$ and $\boldsymbol{D}=\boldsymbol{I}$, then the transmitted symbols $\boldsymbol{s}=[0.25+0.25 j ; 0.25-0.25 j]$ would result in the received signal power reduction by a factor of 0.4062 , while an integer remapping to $\tilde{\boldsymbol{s}}=[1.25+0.25 j,-0.75-0.25 j]$ would bring the factor down to 0.2812 .

\section{ExPANDED CONSTEllation MAPPING}

Let us now introduce the proposed ECM for maximising the received signal power, while still attaining complete FEXT pre-cancellation. Structurally, ECM is comprised of the match entity and of the control entity, both preceding the linear vectoring module of G.fast, with minimal modifications of the current architecture, as seen in Fig. 1.

1) Match Entity: The match entity of Fig. 1 is operated on a per-tone basis. The transmitted symbols $s$ of a particular tone are mapped to a newly formed collection of expanded constellation sets $\mathcal{A}_{e}$, whilst aiming for best matching the FEXT pre-cancellation matrix $\boldsymbol{H}^{-1}$, in order to minimise the received signal power reduction. In a nutshell, the match entity of Fig. 1 determines the best mapping $\tilde{\boldsymbol{s}}$ by solving the optimisation problem of

$$
\tilde{\boldsymbol{s}}=\arg \min _{\boldsymbol{s} \in \mathcal{A}_{e}}\left\|\boldsymbol{H}^{-1} \boldsymbol{D} \boldsymbol{s}\right\|^{2} \quad \text { s.t. } \quad \mathcal{A}_{e}=\left\{\mathcal{A}_{k}^{r_{k}}\right\}_{k=1 \ldots K},
$$

where $\mathcal{A}_{k}^{r_{k}}$ represents the expanded constellation set for the $k$ th transmitted symbol $s_{k}$ and the superscript $r_{k}$ represents the corresponding range of the expanded constellation set. In order to find $\tilde{\boldsymbol{s}}$, we firstly have to perform constellation expansion and then carry out the mathematical optimisation of (2).

Expansion: The construction of the expanded constellation set $\mathcal{A}_{k}^{r_{k}}$ is as follows. Let $\mathcal{A}_{k}^{0}$ be the original constellation set corresponding to $r_{k}=0$, which is normalised within $( \pm 1 \pm j) / 2$. Given any positive integer value of $r_{k}$, the original constellation set $\mathcal{A}_{k}^{0}$ is firstly expanded along the real dimension to arrive at $\mathcal{R}\left(\mathcal{A}_{k}^{r_{k}}\right)=\left\{\mathcal{A}_{k}^{0}, \mathcal{A}_{k}^{0} \pm 1, \cdots \mathcal{A}_{k}^{0} \pm r_{k}\right\}$, which is then further expanded along the imaginary dimension to arrive at $\mathcal{A}_{k}^{r_{k}}=\left\{\mathcal{R}\left(\mathcal{A}_{k}^{r_{k}}\right), \mathcal{R}\left(\mathcal{A}_{k}^{r_{k}}\right) \pm j, \cdots \mathcal{R}\left(\mathcal{A}_{k}^{r_{k}}\right) \pm r_{k} j\right\}$. Repeat the construction of $\mathcal{A}_{k}^{r_{k}}$ for all transmitted symbols, the collection of expanded constellation sets $\mathcal{A}_{e}$ is formed. An example of the above expansion is shown in Fig. 1.

Optimisation: After obtaining $\mathcal{A}_{e}$, optimisation is carried out to solve the problem defined in (2) in order to find $\tilde{\boldsymbol{s}}$. There are numerous ways of solving this problem, but the classic Lattice Aided Search (LAS) algorithms [6] will be used in Section IV. In general, a larger expansion range will result into a better performance, but at the cost of an increased complexity ${ }^{2}$ and an increased Peak to Average Power Ratio

\footnotetext{
${ }^{2}$ The exact complexity is very difficult to quantify, since it depends on the underlying lattice basis, problem space, search strategy, etc. But according to [7], the expected complexity is exalted from a polynomial to an exponential trend, when increasing the size of the search space.
}

(PAPR) of the resultant expanded constellations. In particular, when the expansion tends to infinity and all the original constellation sets are identical, then ECM becomes reminiscent of the vector perturbation concept of [8], which suffers from a high complexity and an unpredictable PAPR.

Detection: Once $\tilde{\boldsymbol{s}}$ is found, it is used instead of $\boldsymbol{s}$ and we transmit $\boldsymbol{x}=\sqrt{1 / \beta_{\min }} \boldsymbol{H}^{-1} \boldsymbol{D} \tilde{\boldsymbol{s}}$ in conjunction with $\beta_{\min }=$ $\mathbb{E}_{\boldsymbol{s}}\left[\left\|\boldsymbol{H}^{-1} \boldsymbol{D} \tilde{\boldsymbol{s}}\right\|^{2}\right]$. Hence, (1) becomes $\tilde{\boldsymbol{y}}=\sqrt{1 / \beta_{\min }} \boldsymbol{D} \tilde{\boldsymbol{s}}+\boldsymbol{n}$. Plausibly, since $\beta_{\min }$ is the minimum value of $\beta$, the received signal power after applying ECM is maximised, while still maintaining complete FEXT pre-cancellation. At the $k$ th NT, the equivalent system model may be written as $\gamma_{k}=\alpha_{k} \tilde{y}_{k}=$ $\tilde{s}_{k}+w_{k}$ in conjunction with $\alpha_{k}=\sqrt{\beta_{\min }} d_{k}^{*} /\left|d_{k}\right|^{2}$, where $d_{k}$ is the $k$ th diagonal entry of $\boldsymbol{D}$ and the superscript $*$ stands for the conjugate operator. Since $\tilde{s}_{k}$ is the integer expansion of $s_{k}$, in order to demodulate $s_{k}, \gamma_{k}$ has to be returned to the original constellation set $\mathcal{A}_{k}^{0}$ by simply applying the modulo operation of $\wedge_{\zeta}$ over the per-dimensional range of $\zeta=[-1 / 2,1 / 2]$. Hence, we have

$$
y_{k}=\wedge_{\zeta}\left(\gamma_{k}\right)=\wedge_{\zeta}\left(s_{k}+w_{k}\right),
$$

where $w_{k}$ is the equivalent AWGN having a zero mean and a variance of $\sigma_{w_{k}}^{2}=\sigma^{2} \beta_{\min } /\left|d_{k}\right|^{2}$.

2) Control Entity: The match entity of Fig. 1 is operated on a per-tone basis, while an extra control entity of Fig. 1 is introduced to determine the specific subset of tones that the match entity are activated. The activation process is described as follows. Firstly, calculate the values of $\beta$ as if linear vectoring was used for all tones. Secondly, sort all these values according to descending order. Finally, apply the match entity on $m_{e}$ out of all $M$ tones associated with the first $m_{e}$ values of $\beta$, where the choice of $m_{e}$ depends on the specific system requirements.

In addition to per-tone based match entity activation, the control entity can also determine how the match entity would be applied. On a particular tone which is activated, the control entity can determine the range of the expanded constellation set for all $K$ transmitted symbols. The control entity may also select a subset of the lines to apply the match entity, resulting into partial or group ECM. Furthermore, the control entity may determine the optimisation methods depending on the affordable complexity. Owing to space limitation, these possibilities are not exploited in this letter.

3) Finite Alphabet Capacity: Let us now consider the modulation-mode-specific finite alphabet capacity of ECM. In this letter, we consider symmetric real and imaginary parts for $\mathcal{A}_{k}^{0}$. Based on (3), the mutual information between the received signal $y_{k}$ and the transmitted symbol $s_{k}$ may be formulated as $I\left(s_{k} ; y_{k}\right)=I\left(s_{k, r} ; y_{k, r}\right)+I\left(s_{k, i} ; y_{k, i}\right)$ due to the independence between the real and the imaginary parts of $s_{k}$ and $y_{k}$. Hence, we only elaborate on the real dimension as an example, when $s_{k, r}$ belongs to a specific constellation $\mathcal{A}_{k, r}^{0}$, where $\mathcal{A}_{k, r}^{0}$ stands for the real part of $\mathcal{A}_{k}^{0}$. Formally, the mutual information $I\left(s_{k, r} ; y_{k, r}\right)$ may be expressed as

$$
\begin{aligned}
& I\left(s_{k, r} ; y_{k, r}\right)= \\
& \max _{p\left(s_{k, r}\right)} \sum_{s_{k, r}} \int_{y_{k, r}} p\left(y_{k, r}, s_{k, r}\right) \log _{2}\left(\frac{p\left(y_{k, r} \mid s_{k, r}\right)}{p\left(y_{k, r}\right)}\right) \mathrm{d} y_{k, r},
\end{aligned}
$$



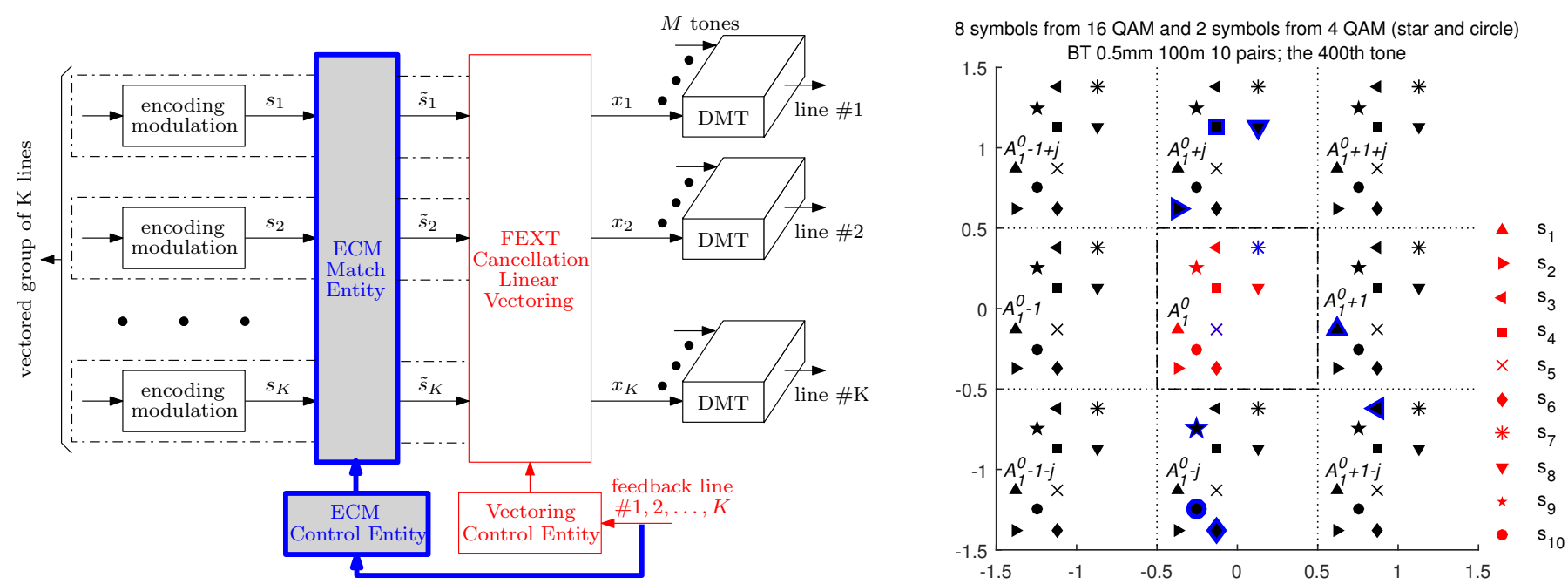

Fig. 1. The transmitter architecture of G.fast with both the match entity and the control entity of ECM (left). An example of ECM on the 400th tone of the BT copper lines having $0.5 \mathrm{~mm}$ width, $100 \mathrm{~m}$ length and 10 pairs (right). In this example, 10 symbols are going to be transmitted with 8 symbols from 16 QAM $\left(s_{1}, \ldots, s_{8}\right)$ and 2 symbols from 4 QAM $\left(s_{9}, s_{10}\right)$. Their original constellation sets may be seen in the center dashed block, e.g. $\mathcal{A}_{1}^{0}$ is the original constellation set for $s_{1}$ in triangle. In addition to original constellation set, expanded constellation set is also constructed with an unity range for each of the 10 symbols, e.g. $\mathcal{A}_{1}^{1}=\left\{\mathcal{A}_{1}^{0}, \mathcal{A}_{1}^{0}+1, \mathcal{A}_{1}^{0}-1, \mathcal{A}_{1}^{0}+j, \mathcal{A}_{1}^{0}+1+j, \mathcal{A}_{1}^{0}-1+j, \mathcal{A}_{1}^{0}-j, \mathcal{A}_{1}^{0}+1-j, \mathcal{A}_{1}^{0}-1-j\right\}$ is the expanded constellation set for $s_{1}$ in triangle. Hence, for each of the 10 symbols, there are a total of 9 positions to map to, e.g. $\left|\mathcal{A}_{1}^{1}\right|=9$, where $|\cdot|$ stands for the cardinality of a set. After applying ECM, 2 symbols remain fixed in position ( $s_{5}$ in cross and $s_{7}$ in asterisk), while 8 symbols are mapped to their respective new positions in larger legends, e.g. $s_{1}$ in triangle is mapped from $\mathcal{A}_{1}^{0}$ to $\mathcal{A}_{1}^{0}+1$.

where the summation in (4) is carried out over $\mathcal{A}_{k, r}^{0}$. Furthermore, (4) is maximised, when $s_{k, r}$ obeys uniform distribution, namely when we have $p\left(s_{k, r}\right)=1 /\left|\mathcal{A}_{k, r}^{0}\right|$. This is because the uniform distribution maximises the entropy for discrete input. After some manipulations, (4) may be further expressed as

$$
\begin{aligned}
& I\left(s_{k, r} ; y_{k, r}\right)= \\
& \log _{2}\left(\left|\mathcal{A}_{k, r}^{0}\right|\right)-\frac{1}{\left|\mathcal{A}_{k, r}^{0}\right|} \sum_{s_{k, r}} \mathbb{E}_{y_{k, r}}\left[\log _{2} \sum_{s_{k, r}^{\prime}} \frac{p\left(y_{k, r} \mid s_{k, r}^{\prime}\right)}{p\left(y_{k, r} \mid s_{k, r}\right)}\right],
\end{aligned}
$$

where numerical evaluation is required to obtain (5) and the likelihood function $p\left(y_{k, r} \mid s_{k, r}\right)$ may be expressed as

$$
p\left(y_{k, r} \mid s_{k, r}\right) \propto \sum_{z \in \mathbb{Z}} e^{-\left(y_{k, r}-s_{k, r}-z\right)^{2} / \sigma_{w_{k}}^{2}}, \quad y_{k, r} \in \zeta .
$$

\section{Numerical Performance}

Let us now provide numerical results for characterising the proposed ECM. For all simulations, we use realistic measurements from 10 pairs of British Telecom (BT) copper lines having $0.5 \mathrm{~mm}$ width and $100 \mathrm{~m}$ length together with 400 tones spanning from $517.5 \mathrm{kHz}$ to $207 \mathrm{MHz}$. We use equal power allocation for all tones and employ the same modulation on all tones and for every lines, where the noise power is set to -100 $\mathrm{dBm}$ per tone. We employ ECM with unity range. Finally, we adopt the Blockwise Korkine Zolotarev (BKZ) algorithm [9] for optimisation in the match entity. Note that, 4096 QAM is considered corresponding to the maximum number of bits defined in the standard, which is 12 .

Fig. 2 shows the received signal power gain in $\mathrm{dB}$ across all 400 tones as a benefit of applying ECM. Observe that a beneficial gain starts to appear from around the 100th tone, which is then dramatically increased to an average (maximum) gain of about $12 \mathrm{~dB}(30 \mathrm{~dB})$ in received signal power.

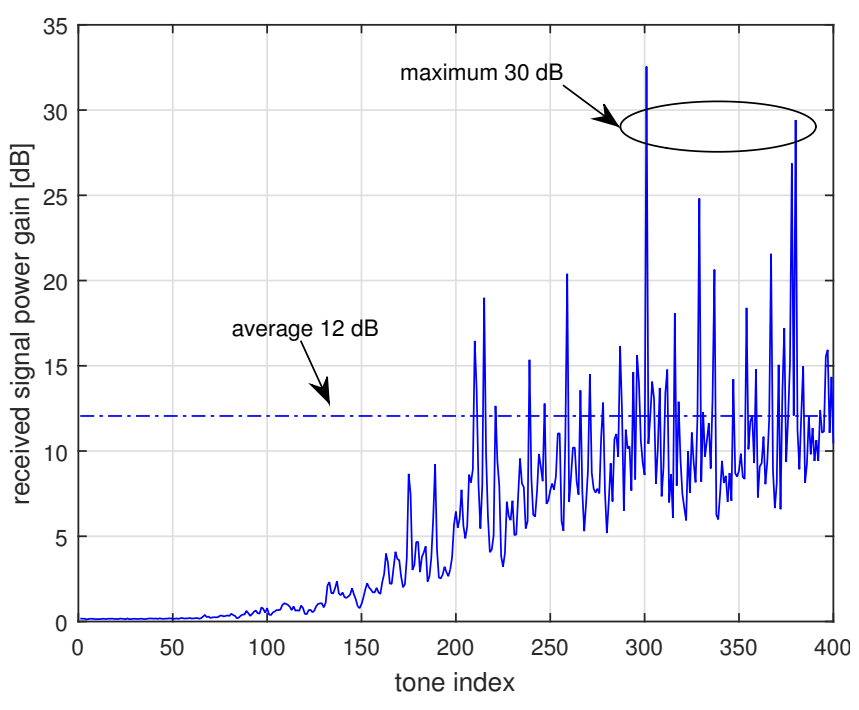

Fig. 2. The received signal power gain in $\mathrm{dB}$ across all 400 tones.

Fig. 3 compares the average symbol error probability of ECM with unity range and of the two benchmarks, i.e. of the linear vectoring and of the non-linear vectoring, for both 16 QAM (solid) and 4096 QAM (dash). It is clear that for 16 QAM, ECM (hollow square) exhibits more than $25 \mathrm{~dB}$ gain in power efficiency over the linear vectoring (diamond) and around $20 \mathrm{~dB}$ gain over the non-linear vectoring (triangle). Substantial gains can also be observed for 4096 QAM. Furthermore, considering the intercept point ' $\mathrm{A}$ ' between ECM with 4096 QAM (12 bits) and linear vectoring with 16 QAM (4 bits), 3-fold gain in bandwidth efficiency is achievable as a benefit of applying ECM. Finally, despite the $5 \mathrm{~dB}$ in power efficiency reduction of ECM observed under a per-line power constraint (filled square) for both modulations, a substantial gain still exists. 


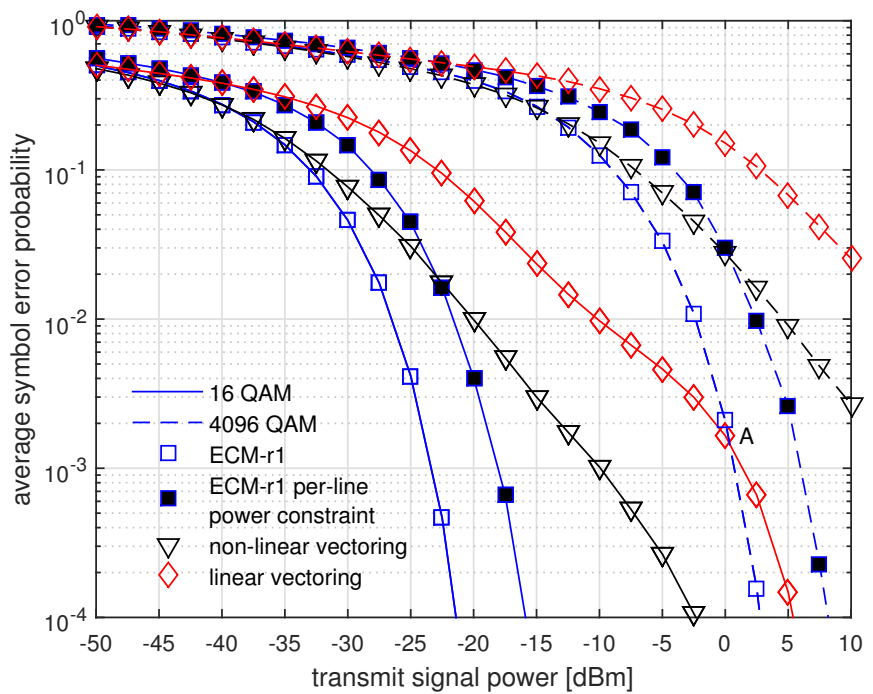

Fig. 3. The comparisons of the average symbol error probability between ECM and the two benchmarks, for both 16 QAM and 4096 QAM.

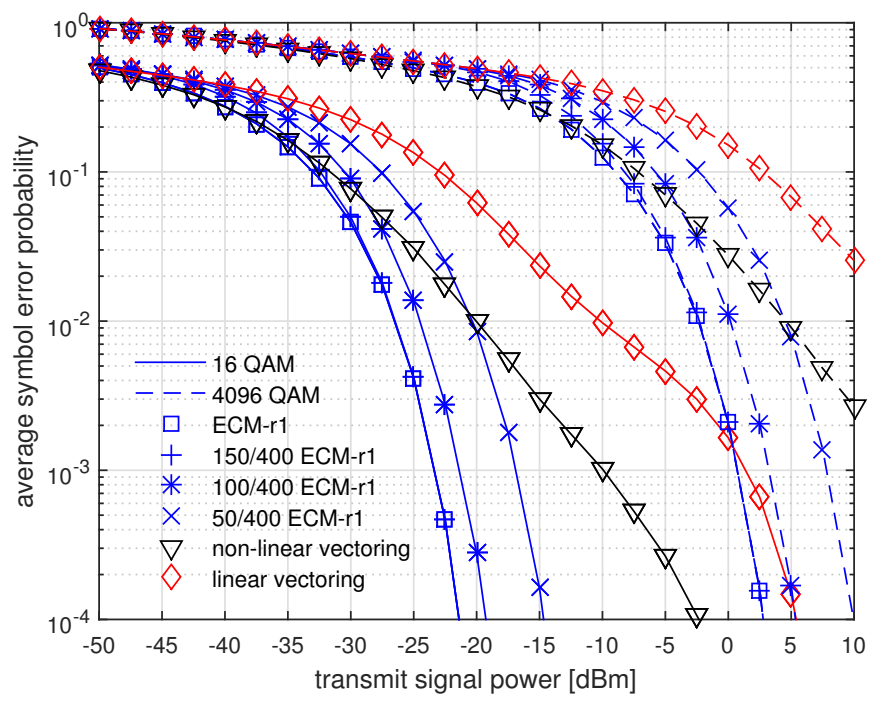

Fig. 4. The average symbol error probability of ECM with partial toneactivation of the match entity, for both 16 QAM and 4096 QAM.

Fig. 4 shows the average symbol error probability of ECM with unity range relying on partial tone-activation of the match entity. Three tone-activation settings were included, namely 50/400 tones (cross), 100/400 tones (asterisk) and 150/400 tones (plus). It is clear that for both 16 QAM (solid) and 4096 QAM (dash), activating 150/400 tones exhibits negligible performance degradation, when compared to full activation (square), while activating 50/400 tones still maintains a substantial gain over both the linear vectoring (diamond) and over the non-linear vectoring (triangle).

Fig. 5 shows the modulation-mode-specific finite alphabet capacity of ECM with unity range when compared to that of the linear vectoring, for both 16 QAM (solid) and 4096 QAM (dash). Furthermore, the capacity achieved by dirty paper coding is used as the upper bound. It is clear that ECM (square) is capable of achieving a significantly higher finite alphabet capacity than that of the linear vectoring (diamond) for both modulations. Furthermore, the finite alphabet capacity of ECM

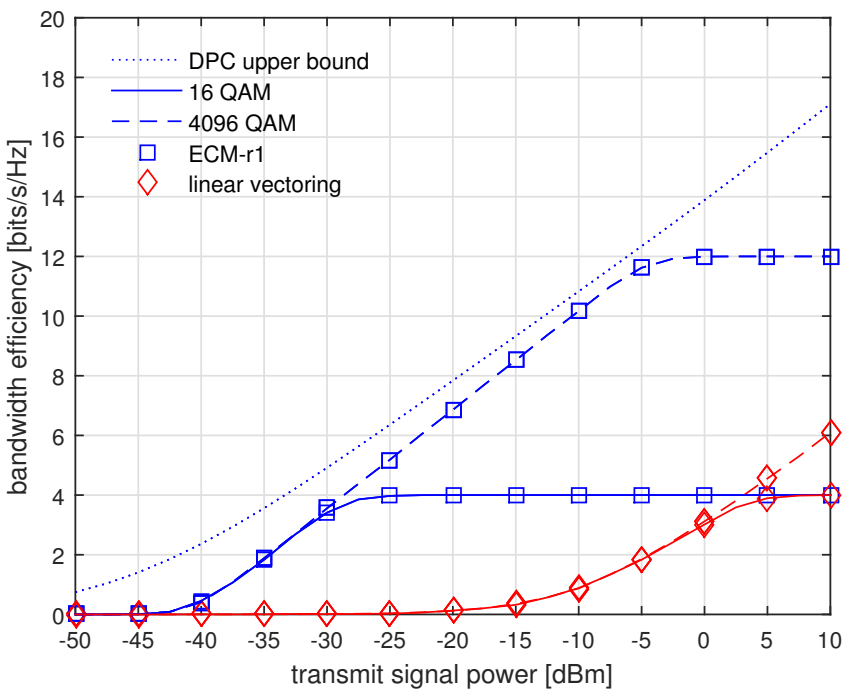

Fig. 5. The comparisons of achievable capacity between ECM and the linear vectoring as well as the dirty paper coding as upper bound.

for 4096 QAM approaches the upper bound capacity (dot) before reaching its maximum, demonstrating the optimality of ECM.

\section{CONCLUSIONS}

In this letter, we proposed the novel ECM for enhanced FEXT cancellation in G.fast. We found that ECM is capable of offering substantial gains over the operational benckmarks. Practically, ECM imposes a minimal structural change at the G.fast transmitter with near-zero-modifications at the receiver. It also exhibits beneficial per-tone based reconfigurability and backward compatibility, since it constitutes an enhancement preceding the linear vectoring module of G.fast, which can be simply turned off to revert back to the current solution.

\section{REFERENCES}

[1] M. Timmers, M. Guenach, C. Nuzman, and J. Maes, "G.fast: evolving the copper access network," IEEE Communications Magazine, vol. 51, no. 8, pp. 74-79, 2013.

[2] J. Maes and C. Nuzman, "The past, present, and future of copper access," Bell Labs Technical Journal, vol. 20, pp. 1-10, 2015.

[3] R. Cendrillon, G. Ginis, E. V. den Bogaert, and M. Moonen, "A nearoptimal linear crosstalk precoder for downstream VDSL," IEEE Transactions on Communications, vol. 55, no. 5, pp. 860-863, 2007.

[4] G. Ginis and J. Cioffi, "Vectored transmission for digital subscriber line systems," IEEE Journal on Selected Areas in Communications, vol. 20, no. 5, pp. 1085-1104, 2002.

[5] C. Peel, B. Hochwald, and A. Swindlehurst, "A vector-perturbation technique for near-capacity multiantenna multiuser communication-part I: channel inversion and regularization," IEEE Transactions on Communications, vol. 53, no. 1, pp. 195-202, 2005.

[6] E. Agrell, T. Eriksson, A. Vardy, and K. Zeger, "Closest point search in lattices," IEEE Transactions on Information Theory, vol. 48, no. 8, pp. 2201-2214, 2002.

[7] B. Hassibi and H. Vikalo, "On the sphere-decoding algorithm i. expected complexity," IEEE Transactions on Signal Processing, vol. 53, no. 8, pp. 2806-2818, 2005.

[8] B. Hochwald, C. Peel, and A. Swindlehurst, "A vector-perturbation technique for near-capacity multiantenna multiuser communication-part II: perturbation," IEEE Transactions on Communications, vol. 53, no. 3, pp. 537-544, 2005.

[9] C. P. Schnorr and M. Euchner, "Lattice basis reduction: Improved practical algorithms and solving subset sum problems," Mathematical Programming, vol. 66, no. 1, pp. 181-199, 1994. 